\title{
A Bioinspired Tilt Sensor Model with Adaptive Gain and Enhanced Sensitivity
}

\author{
Lijun Liu ${ }^{1,2}$ and Ying Lei ${ }^{2}$ \\ ${ }^{1}$ Department of Mechanical and Electrical Engineering, Xiamen University, Xiamen 361005, China \\ ${ }^{2}$ Department of Civil Engineering, Xiamen University, Xiamen 361005, China \\ Correspondence should be addressed to Ying Lei; ylei@xmu.edu.cn
}

Received 27 January 2014; Accepted 8 April 2014; Published 27 April 2014

Academic Editor: Ting-Hua Yi

Copyright (c) 2014 L. Liu and Y. Lei. This is an open access article distributed under the Creative Commons Attribution License, which permits unrestricted use, distribution, and reproduction in any medium, provided the original work is properly cited.

\begin{abstract}
Although various types of tilt sensors have been proposed in the past decade, it is still essential to develop rugged, cheap, simplestructured tilt sensors with wide measuring range and high sensitivities for efficient monitoring of infrastructures and early warning of natural disasters. It has been investigated that stereocilia in some fishes' inner ear organs are the basic sensory units of nature's inertial sensors and are highly sensitive over broad dynamic range because of a combination of adaptation and negative stiffness mechanisms. In this paper, a bioinspired tilt sensor model is proposed that mimics the mechanism of stereocilia in adaptive signal amplification to mechanical stimuli, leading to high sensitivity to weak input and low sensitivity to high input, thus expanding the dynamic range through adaptive amplification. The negative stiffness mechanism is implemented by magnet forces. The tilt motion is measured by the strain gauge at the end of the flexible cantilever beam element in the model. Measurements of both static and dynamics tilt motion are investigated. Numerical simulation results are used to demonstrate the capability of the proposed model for the measurements of tilt motions with adaptive amplification and enhanced sensitivity.
\end{abstract}

\section{Introduction}

Tilt sensors, also known as inclinometers, are used to measure the angular deflection of an object against a reference plane or line. Tilt angle measurements have wide applications in many fields $[1,2]$. In the past decade, various types of tilt sensors were reported based on the principles and implementations such as resistive, capacitive, inductive, magnetic, optical, mechanics, thermodynamics, fiber-optic, and electrolyte [2-8]. However, with the measurement tilt increases, the sensitivity of a tilt sensor usually decreases seriously; that is, the larger tilt it measures, the lower sensitivity it becomes. Therefore, how to improve the sensitivity of tilt sensor is the technical difficulty to achieve an accurate and wide range measurement in tilt [9]. It is still essential to develop rugged, cheap, simple-structured tilt sensors with wide measuring range and high sensitivities for early warning of natural disasters; for example, it is still changing to develop tilt with high-sensitive and wide range measurement for monitoring geological disasters, such as landslides $[10,11]$. Also, it is important to measure the dynamic rotational angle responses of buildings, bridges, and other civil infrastructures, but it is still difficult to accurately measure these small dynamic rotational responses in practice. Commonly, tilt or inclination has been mathematically derived from another measurement response; however, there is inherent error in any indirect measurements [12-14]. Therefore, there is also a need to develop tilt sensors with high-precision to small dynamic rotational angles for efficient health monitoring of infrastructures [15-19].

In marine biology, some experiments have shown that some fishes can detect very weak motion with their inner ear organs [20,21]. Stereocilia in these inner ear organs are the basic sensory units of nature's inertial sensors and are highly sensitive over broad dynamic range because they display adaptive signal amplification to mechanical stimuli, leading to high sensitivity to weak input and low sensitivity to high input, thus expanding the dynamic range through adaptive amplification [21, 22]. Some researchers have explored that the high sensitivity that is maintained by stereocilia is hypothesized to exist due to a combination of adaptation and negative stiffness mechanisms, which shift the region of 
highest sensitivity toward the active operation range of the stereocilia $[23,24]$. Based on such adaptation and negative stiffness mechanisms, some bioinspired sensor model has been developed $[25,26]$. The authors also have investigated the mechanism of a hair cell bioinspired sensor with ultrasensitivity to weak and low frequency vibration signals [27].

In this paper, based on the combination of adaptation and negative stiffness mechanisms of the stereocilia in some fishes' inner ear organs, a bioinspired mechanical model of tilt sensor with adaptive gain and enhanced sensitivity is proposed. The negative stiffness mechanism is implemented by a magnet pair attached to the top of a fixed cantilever beam element and a rigid bar, respectively, which emulates the negative resistance of the tip-link due to the transient stiffness softening by the gating ion channel [24]. The tilt motion is measured by the strain gauge at the end of the cantilever beam element. Measurement of the static tilt motion is first studied. Then, the measurement of the dynamic tilt motion is investigated. Both numerical simulation results are used to demonstrate the capability of the proposed model for the measurements of tilt motions with adaptive amplification and high sensitivity.

\section{The Bioinspired Mechanical Model of the Tilt Sensor}

As shown in Figure 1(a), the proposed model consists of a fix light weight flexible cantilever beam (right) with a concentrated mass at the tip, which mimics the stereocilia bundle and the otolith in some fish's inner ear organs, and a fix rigid bar (left). The bending stiffness of the beam in the $x-z$ plan is much larger than that in the $x-y$ plan, so the beam is assumed to be deflected in the $x-y$ plan. To generate the stiffness softening by the gating spring [27], a magnet pair facing the same pole is attached to the top of the beam element and rigid bar, respectively, to generate a repulsive force against each other. When the base is not tilted in the $x-y$ plan, the magnet pair is perfectly aligned such that there is no net force to bend the beam element in the $x-y$ plan; this represents a closed ion channel without gating. However, when the base is tilted in the $x-y$ plan as shown in Figure $1(\mathrm{~b})$, there is a force at the top mass due to the fact that gravity increases in horizontal direction of the $x-y$ plan; the beam element deflects in the $x-y$ plan accordingly. The repulsive magnetic force enhances the bending movement of the beam element in the plan until the repulsive force is in equilibrium with the elastic restoring force. This mimics the negative resistance of the stereocilia bundle in response to the miniscule stimuli during the channel opening by the gating.

The magnet pair generates repulsive forces that are inversely proportional to the distance squared [28]; that is,

$$
\begin{aligned}
\mathbf{F}= & \frac{J \times J^{\prime}}{4 \times \pi \times \mu_{0}} \\
& \times \sum_{i=0}^{1} \sum_{j=0}^{1} \sum_{k=0}^{1} \sum_{l=0}^{1} \sum_{p=0}^{1} \sum_{q=0}^{1}(-1)^{i+j+k+l+p+q} \varphi\left(U_{i j}, V_{k l}, W_{p q}, r\right),
\end{aligned}
$$

\begin{tabular}{|c|c|c|}
\hline Item & Value & Definition \\
\hline$l$ & $0.1 \mathrm{~m}$ & Length of the beam element \\
\hline E & $9 \times 10^{5} \mathrm{~N} / \mathrm{m}^{2}$ & $\begin{array}{l}\text { Young's modulus of beam } \\
\text { element }\end{array}$ \\
\hline$I_{z}$ & $6.36 \times 10^{-11} \mathrm{~m}^{4}$ & $\begin{array}{l}\text { Moment of inertia in } z \\
\text { direction of beam element }\end{array}$ \\
\hline$I_{y}$ & inf & $\begin{array}{l}\text { Moment of inertia in } y \\
\text { direction of beam element }\end{array}$ \\
\hline$m$ & $0.002 \mathrm{~kg}$ & Tip mass \\
\hline$\zeta$ & 0.865 & $\begin{array}{l}\text { Damping ratio of model, } \\
\text { the otolith and hair cells are } \\
\text { surrounded by endolymph, } \\
\text { the damping is simplified as } \\
\text { a large value viscous } \\
\text { damping }\end{array}$ \\
\hline$z_{0}$ & $0.02 \mathrm{~m}$ & $\begin{array}{l}\text { The relative coordinate of } \\
\text { the two magnets in } z \\
\text { direction }\end{array}$ \\
\hline
\end{tabular}

TABLE 1: Parameters of mechanical model.

where $\mathbf{F}$ is the vector of magnet force, $\mu_{0}$ is the permeability of vacuum,

$$
\mu_{0}=4 \pi \times 10^{-7} \mathrm{~N} / \mathrm{A}^{2}
$$

$J$ and $J^{\prime}$ are the magnetizations of the two magnets on the top of the rigid bar and the flexible beam, respectively. $\varphi$ is a vector with the component of $\varphi_{y}$ and $\varphi_{x}$ as

$$
\begin{aligned}
& \varphi_{y}(U, V, W, r)= \frac{\left(U^{2}-W^{2}\right)}{2} \ln (r-V) \\
&+U V \ln (r-U)+U W \operatorname{tg}^{-1}\left(\frac{U V}{W r}\right)+\frac{1}{2} V r \\
& \varphi_{x}(U, V, W, r)= \frac{\left(V^{2}-W^{2}\right)}{2} \ln (r-U) \\
&+U V \ln (r-V)+V W \operatorname{tg}^{-1}\left(\frac{U V}{W r}\right)+\frac{1}{2} U r, \\
& U_{i j}=x_{0}+(-1)^{j} A-(-1)^{i} a ; \\
& V_{i j}=y_{0}+(-1)^{l} B-(-1)^{k} b ; \\
& W_{p q}=z_{0}+(-1)^{q} C-(-1)^{p} c \\
& r=\sqrt{U_{i j}^{2}+V_{k l}^{2}+W_{p q}^{2}}
\end{aligned}
$$

in which $\left(x_{0}, y_{0}, z_{0}\right)$ is the relative coordinates between the two centers of the magnet pair. $A, B, C$ and $a, b, c$ are the size of the two magnets in the $x, y$ and directions, respectively.

In the model, the sizes of the two magnets are selected as $2 A=2 a=0.006 \mathrm{~m}, 2 B=2 b=0.006 \mathrm{~m}, 2 C=2 c=$ $0.006 \mathrm{~m}$, and the two magnetizations are $J=J^{\prime}=0.38$. Other parameters are shown in Table 1. 


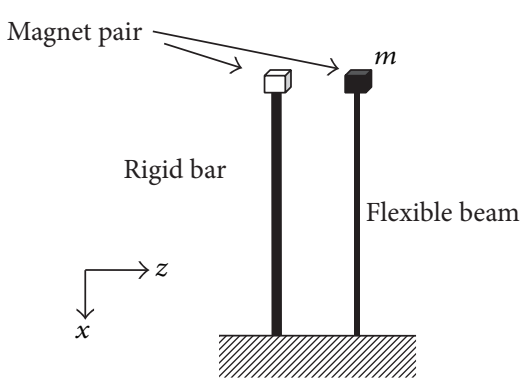

(a)

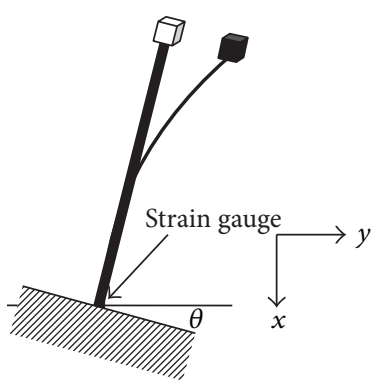

(b)

FIgURE 1: The bioinspired model of the tilt sensor.

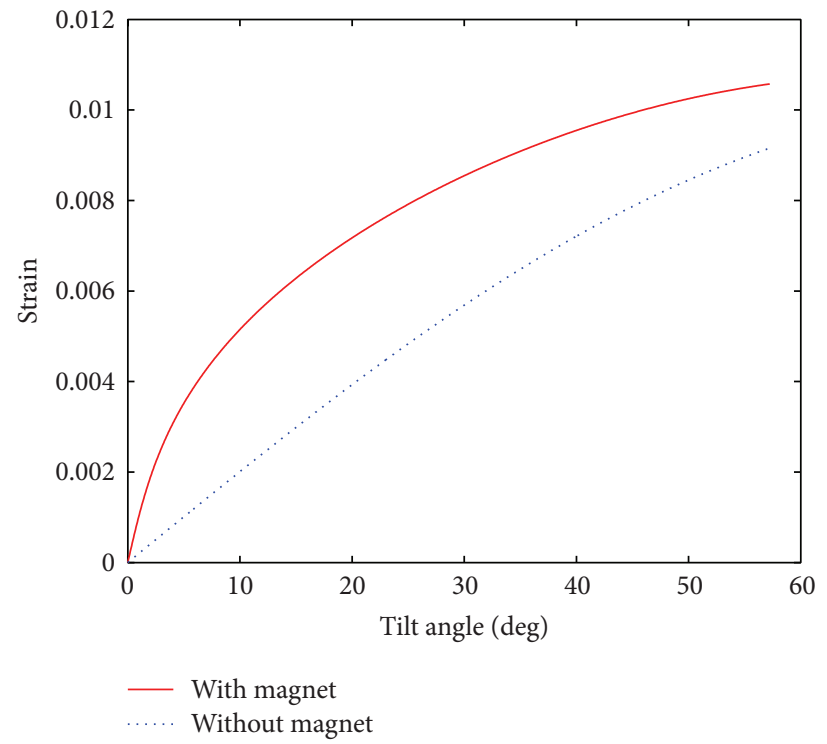

(a) Comparisons of strain under static tilt angle

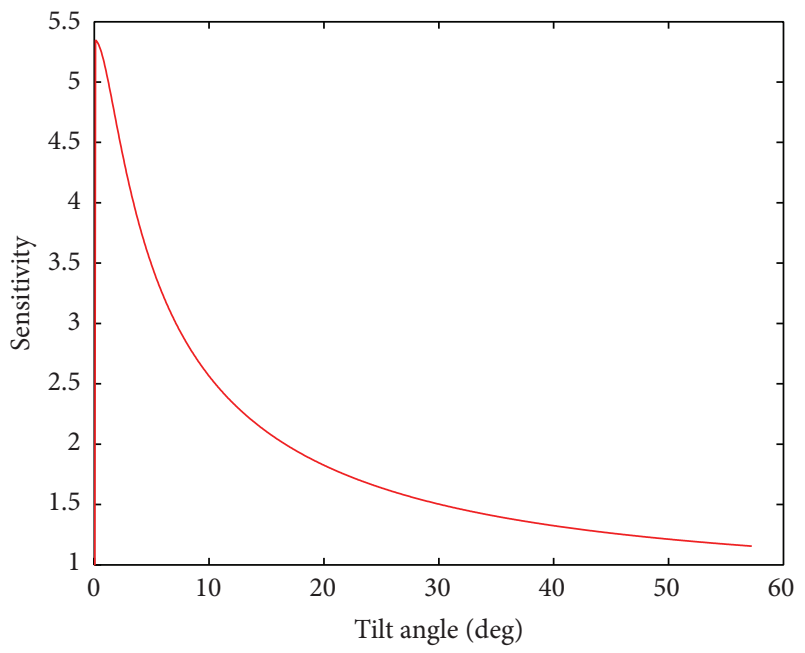

(b) Sensitivity under static tilt

FIGURE 2: Measurement results of the model under static tilt angle.

\section{Measurements of Tilt Motions}

The proposed model in Figure 1(a) is used to measure the tile motion $\theta$ of the base in Figure 1(b). The tilt motion is measured by the strain gauge at the end of the flexible cantilever beam element in the model as shown in Figure 1(b).

3.1. Measurements of Static Tilt Motions. A static tilt angle is applied at the base shown in Figure 1(b). The equations of motion for the two models with magnet and without magnet can be obtained as (4) and (5), respectively,

$$
\begin{gathered}
k \delta=\left(m g+F_{x}\right) \sin \left(\theta+\frac{\delta}{l}\right)+F_{y} \cos \left(\theta+\frac{\delta}{l}\right) \\
k \delta=m g \sin \left(\theta+\frac{\delta}{l}\right)
\end{gathered}
$$

in which $\delta$ is the vertical deflection at the tip of the beam, $k$ is its stiffness, and $F_{x}$ and $F_{y}$ are the components of the magnetic force in the $x$ and $y$ direction, respectively, which are determined by (1)-(3).

To examine the adaptive amplification of the model with magnet, the base is tilted angle $\theta$ from 0 to 60 degree with an interval of 0.01 degree. Figure 2(a) shows the comparisons of strain at the bottom of beam element subject to tilt angle $\theta$ with and without the magnetic force. It is shown that the measured strains in model with magnet are more sensitive to small tilt angles than those in the model without magnet. The amplification sensitivity is defined as the ratio of strain of model with magnet and that of model without magnet. As shown in Figure 2(b), the amplification sensitivity is high for small tilt angles and the sensitivities decrease for larger tilt angles. Therefore, the model has adaptive amplification with high sensitivity to slight tilt motion and low sensitivity to large tilt motion due to the negative stiffness contributed by magnet force.

3.2. Measurements of Dynamic Rotational Motions. The two mechanical models are subject to the dynamic rotational motion $\boldsymbol{\theta}(t)$ of the base. Then, the equations of motion for two 


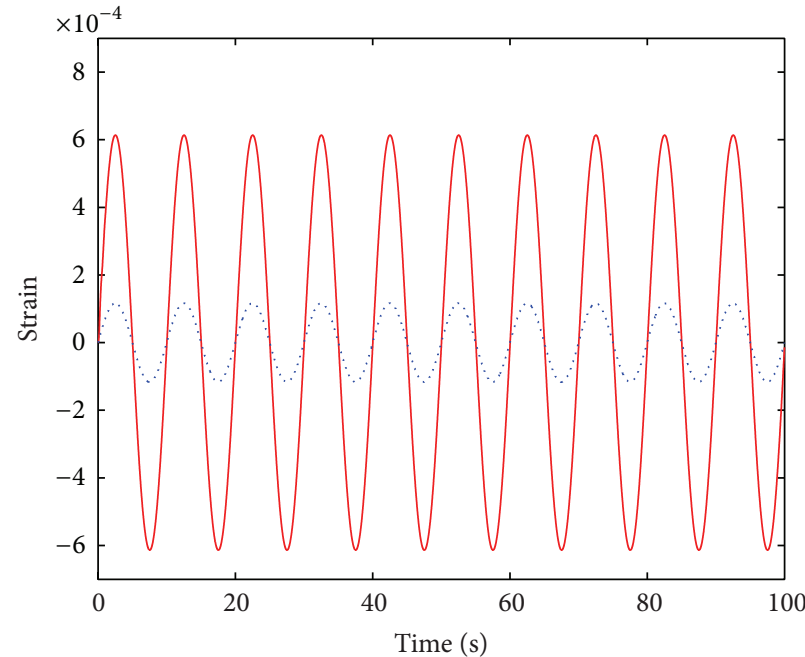

— With magnet ...... Without magnet

(a) $\theta_{0}=0.57^{\circ}$

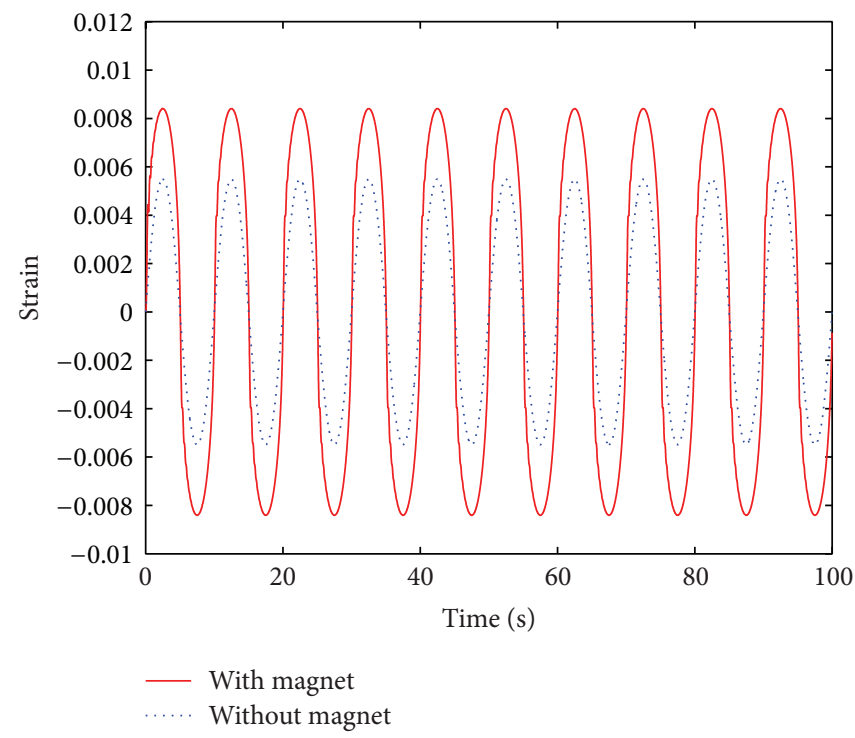

(c) $\theta_{0}=28.5^{\circ}$

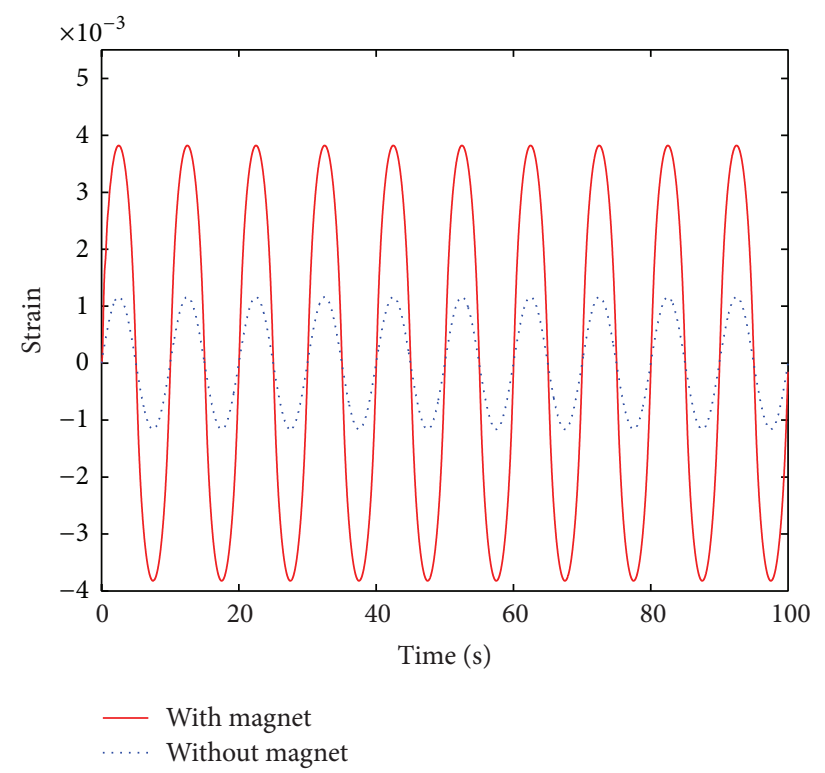

(b) $\theta_{0}=5.7^{\circ}$

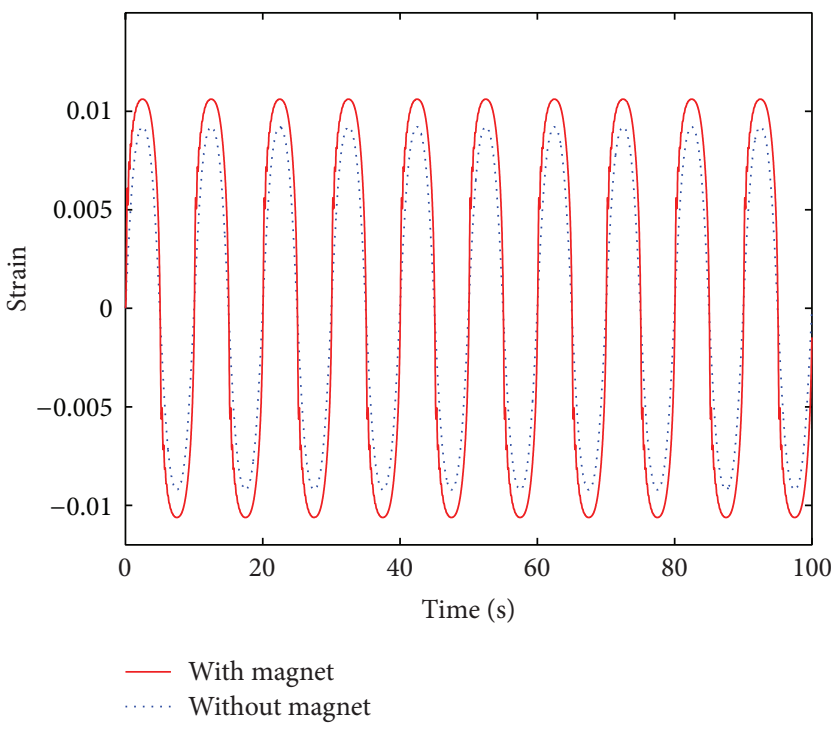

(d) $\theta_{0}=57^{\circ}$

FIGURE 3: Time histories of strains under dynamic base rotations with varying amplitudes.

models with magnet and without magnet can be obtained as (6)

$$
\begin{gathered}
k \delta(t)=-m \ddot{\delta}(t)-c \dot{\delta}(t)-m \ddot{\boldsymbol{\theta}}(t) \\
+\left(m g+F_{x}\right) \sin \left[\boldsymbol{\theta}(t)+\frac{\delta(t)}{l}\right] \\
+F_{y}\left[\cos \boldsymbol{\theta}(t)+\frac{\delta(t)}{l}\right] \\
k \delta(t)=-m \ddot{\delta}(t)-c \dot{\delta}(t)-m i \ddot{\boldsymbol{\theta}}(t)+m g \sin \left[\boldsymbol{\theta}(t)+\frac{\delta(t)}{l}\right],
\end{gathered}
$$

where $\ddot{\boldsymbol{\theta}}(t), \dot{\boldsymbol{\theta}}(t)$, and $\boldsymbol{\theta}(t)$ are the angular acceleration, angular velocity, and angular displacement of tilt motion, respectively, and $c$ is the viscous damping as shown in Table 1.

\subsubsection{Measurements of Dynamic Rotational Motions with} Varying Amplitudes. To examine the adaptive amplification of the model with magnet to the rotational motion with varying amplitudes, it is assumed that the base has the sinusoidal rotation motion; that is, $\boldsymbol{\theta}(t)=\theta_{0} \sin \omega \mathrm{t}$. The amplitude of rotational angle $\theta_{0}$ ranges from 0 to 60 degrees.

Figures $3(a)-3(d)$ show the time history of the strain at the bottom of flexible beam under base rotation motion with frequency equal to $0.1 \mathrm{~Hz}$ and with different amplitude $\theta_{0}$. 


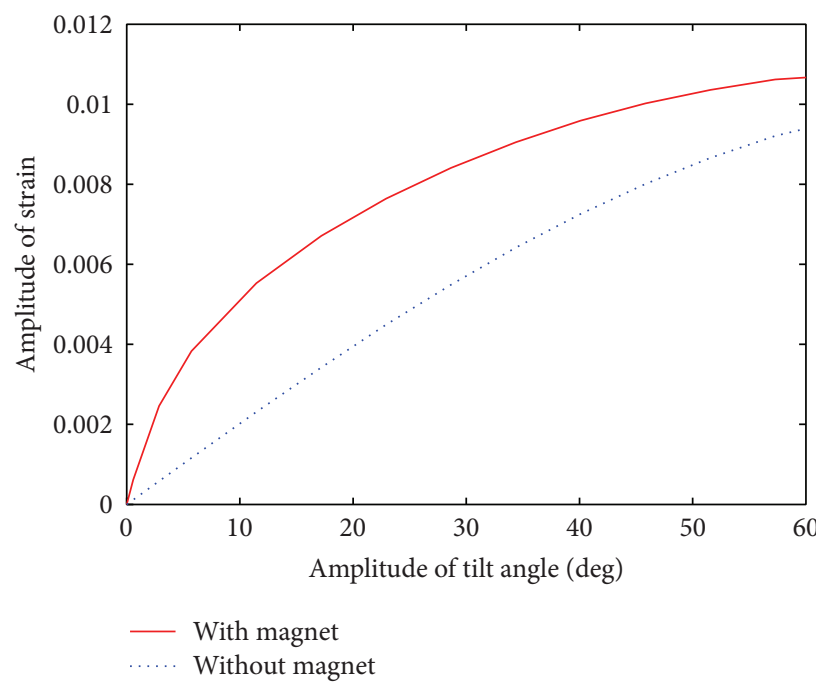

(a) Comparisons of strain amplitudes under dynamic tilt motion

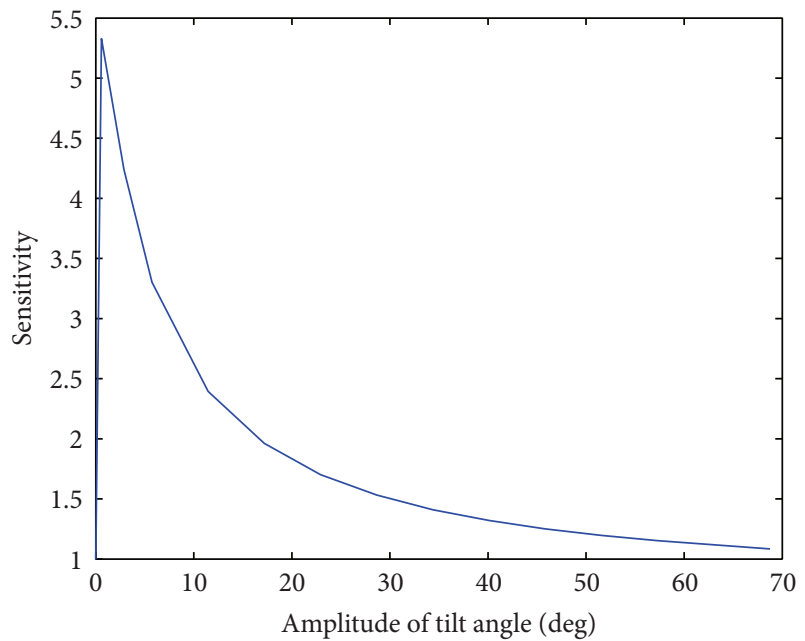

(b) Sensitivity under dynamic tilt motion

FIGURE 4: Measurement results of the model under dynamic tilt motion with varying amplitudes.

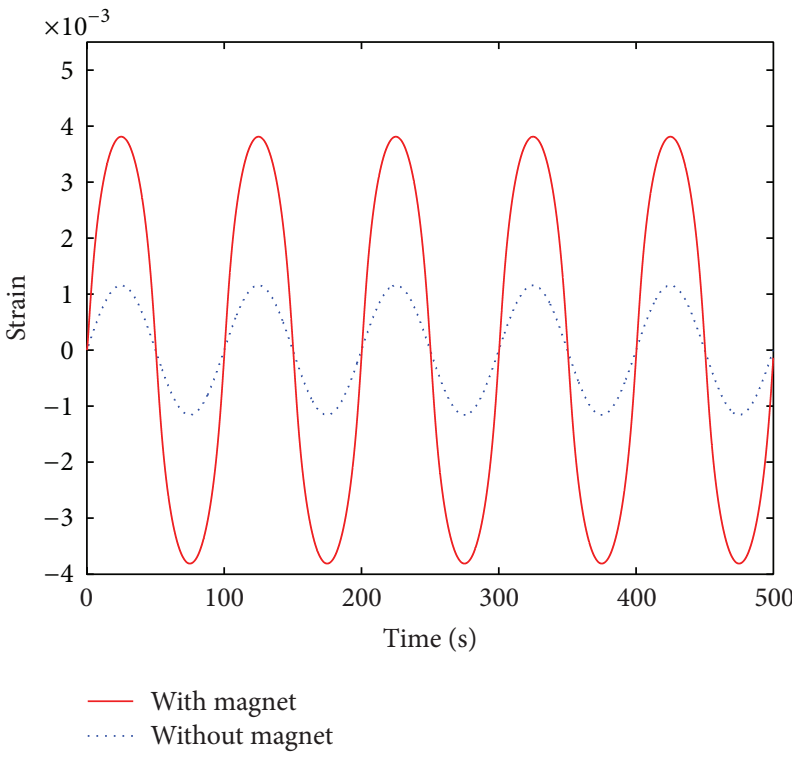

(a) $\omega / \omega_{n}=0.002$

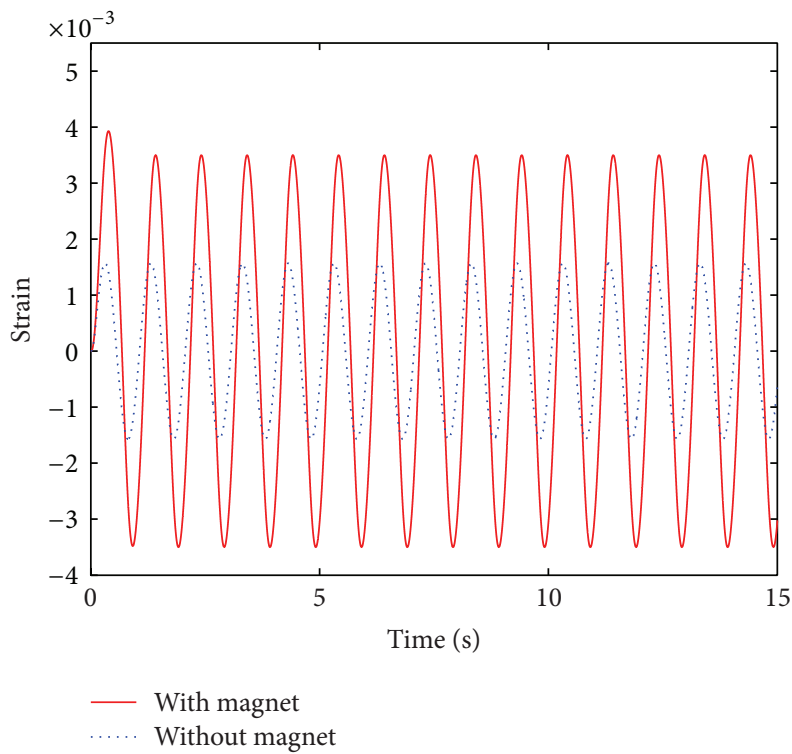

(b) $\omega / \omega_{n}=0.2$

FIGURE 5: Time histories of strains under dynamic base rotations with varying frequencies.

Figure 4(a) shows the comparisons of the amplitudes of strain at the bottom of the flexible beam in two models with and without magnets under dynamic base tilt motions with varying amplitudes $\theta_{0}$.

It is shown that the measured strains in model with magnet are more sensitive to slight tilt motion than those in the model without magnet. The amplification sensitivity is defined as the ratio of amplitude of strain of model with magnet and that of model without magnet. As shown in Figure 4(b), the amplification sensitivity is in highly rising tendency for small amplitude of base rotation angles and is in descent tendency for large amplitude of base rotation angles. This confirms the adaptive amplification capability of the proposed model due to the negative stiffness effect by the magnet forces.

3.2.2. Measurements of Dynamic Rotational Motions with Varying Frequencies. The two models with magnet and without magnet are subject to dynamic base sinusoidal rotational motion with varying frequencies. The amplitude of rotational displacement $\theta_{0}$ is assumed as $0.1 \mathrm{rad}\left(5.7^{\circ}\right)$. Figures 5(a)5(b) compare the time histories of the strain responses at 


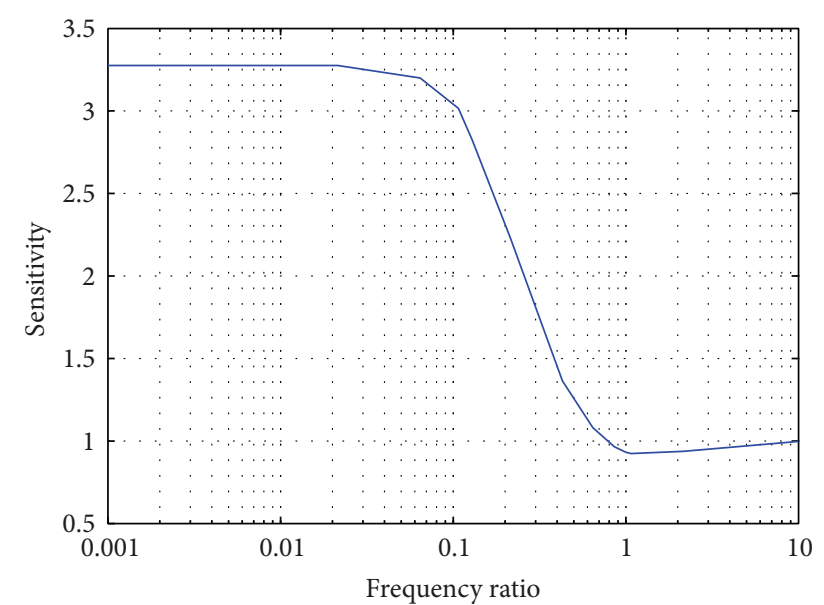

FIGURE 6: The amplification sensitivities for base rotational motion with varying frequencies.

the bottom of the flexible beam in the two models subject to dynamic base sinusoidal rotational motions with varying frequency ratios $\omega / \omega_{n}$, in which $\omega_{n}$ is the natural frequency of the model.

The amplification sensitivities defined under dynamic base rotational motions with varying frequencies are shown in Figure 6. It is clear that the amplification sensitivities are high for base rotational motions with low frequency $(0 \leq$ $\left.\omega / \omega_{n} \leq 0.6\right)$ than those for base rotational motions with high frequency $\left(\omega / \omega_{n}>0.6\right)$. Therefore, it is demonstrated that proposed model is more sensitive to low frequency tilt motion due to the negative stiffness effect by the magnet forces.

\section{Conclusions}

In this paper, based on the mechanisms of adaptation and negative stiffness of the stereocilia in some fishes' inner ear organs, a bioinspired mechanical model of tilt sensor with adaptive gain and enhanced sensitivity is proposed. The negative stiffness effect is implemented by magnet forces and tilt motion can be measured by the strain at the end of flexible cantilever beam element in the model. Numerical simulation results of the measurements of the static and dynamic tilt motions have demonstrated that the proposed tilt model is more sensitive to slight and low frequency tilt motion. Therefore, the proposed tilt model has the capability of adaptive gain and enhanced sensitivity.

The proposed model can be used for the design of bioinspired tilt sensors with adaptive amplification and high sensitivity. More investigations on practical implementation issues of the design of such tilt sensors with small sizes by Micro-Electro-Mechanical System (MEMS) are necessary.

\section{Conflict of Interests}

The authors declare that there is no conflict of interests regarding the publication of this paper.

\section{Acknowledgments}

This work is sponsored by the Natural Science Foundation of Fujian Province of China through the Grant no. 51308482 and by the Science and Technology Key Project of Fujian Province of China through the Grant no. 2013Y0079. The authors thank Professor Y. F. Zhang of University of Maryland for his great contributions to the research project.

\section{References}

[1] S. S. Zhao, J. Zhang C, L. Hou, J. Bai, and G. G. Yang, "Optical tilt sensor with direct intensity-modulated scheme," Optical Engineering, vol. 50, no. 11, Article ID 114405, pp. 1-5, 2011.

[2] J. S. Baji, D. Z. Stupar, L. M. Manojlovi, M. P. Slankamena, and M. B. Zivanova, "A simple, low-cost, high-sensitivity fiber-optic tilt sensor," Sensors and Actuators A, vol. 185, pp. 33-38, 2012.

[3] C. H. Lin and S. M. Kuo, "Micro-impedance inclinometer with wide-angle measuring capability and no damping effect," Sensors and Actuators A: Physical, vol. 143, no. 1, pp. 113-119, 2008.

[4] B. Andò, A. Ascia, and S. Baglio, "A ferrofluidic inclinometer in the resonant configuration," IEEE Transactions on Instrumentation and Measurement, vol. 59, no. 3, pp. 558-564, 2010.

[5] H. Bao, X. Dong, C. Zhao, L.-Y. Shao, C. C. Chan, and P. Shum, "Temperature-insensitive FBG tilt sensor with a large measurement range," Optics Communications, vol. 283, no. 6, pp. 968-970, 2010.

[6] M. Norgia, I. Boniolo, M. Tanelli, S. M. Savaresi, and C. Svelto, "Optical sensors for real-time measurement of motorcycle tilt angle," IEEE Transactions on Instrumentation and Measurement, vol. 58, no. 5, pp. 1640-1649, 2009.

[7] L. M. N. Amaral, O. Frazão, J. L. Santos, and A. B. Lobo Ribeiro, "Fiber-optic inclinometer based on taper Michelson interferometer," IEEE Sensors Journal, vol. 11, no. 9, pp. 1811-1814, 2011.

[8] J. C. Choi, Y. C. Choi, J. K. Lee, H. R. Kim, and S. H. Kong, "A dual-axis tilt sensor using electrolyte," AIP Conference Proceedings, vol. 1476, pp. 74-78, 2012.

[9] W. Su and J. Q. Fu, “The study of variable sensitivity in dual-axis tilt sensor," Procedia Engineering, vol. 29, pp. 2605-2609, 2011.

[10] T. Uchimura, I. Towhata, T. T. L. Anh et al., "Simple monitoring method for precaution of landslides watching tilting and water contents on slopes surface," Landslides, vol. 7, no. 3, pp. 351-357, 2010.

[11] H. Kao, C. Kan, R. Chen et al., "Locating, monitoring, and characterizing typhoon-linduced landslides with real-time seismic signals," Landslides, vol. 9, pp. 557-563, 2012.

[12] Y. Lei, Y. Jiang, and Z. Xu, "Structural damage detection with limited input and output measurement signals," Mechanical Systems and Signal Processing, vol. 28, pp. 229-243, 2012.

[13] T.-H. Yi, H.-N. Li, and M. Gu, "Optimal sensor placement for health monitoring of high-rise structure based on genetic algorithm," Mathematical Problems in Engineering, vol. 2011, Article ID 395101, 12 pages, 2011.

[14] G. D. Zhou and T. H. Yi, "Recent developments on wireless sensor networks technology for bridge health monitoring," Mathematical Problems in Engineering, vol. 2013, Article ID 947867, 33 pages, 2013.

[15] J. Rungamornrat and P. Tangnovarad, "Analysis of linearly elastic inextensible frames undergoing large displacement and 
rotation," Mathematical Problems in Engineering, vol. 2011, Article ID 592958, 37 pages, 2011.

[16] S. D. Glaser, M. Li, M. L. Wang, J. Ou, and J. Lynch, "Sensor technology innovation for the advancement of structural health monitoring: a strategic program of US-China research for the next decade," Smart Structures and Systems, vol. 3, no. 2, pp. 221244, 2007.

[17] T.-H. Yi, H.-N. Li, and M. Gu, "Optimal sensor placement for structural health monitoring based on multiple optimization strategies," Structural Design of Tall and Special Buildings, vol. 20, no. 7, pp. 881-900, 2011.

[18] Z. W. Chen, Y. L. Xu, and X. M. Wang, "SHMS-based fatigue reliability analysis of multiloading suspension bridges," Journal of Structural Engineering, vol. 138, no. 3, pp. 1-10, 2012.

[19] Z. W. Chen, Y. L. Xu, Y. Xia, Q. Li, and K. Y. Wong, "Fatigue analysis of long-span suspension bridges under multiple loading: case study," Engineering Structures, vol. 33, no. 12, pp. 32463256, 2011.

[20] N. Popper, R. R. Fay, C. Platt, and O. Sand, "Sound detection mechanisms and capabilities of teleost fishes," in Sensory Processing in Aquatic Environments, S. Collin and N. Marshall, Eds., pp. 3-38, Springer, New York, NY, USA, 2003.

[21] A. N. Popper and R. R. Fay, "Rethinking sound detection by fishes," Hearing Research, vol. 273, no. 1-2, pp. 25-36, 2011.

[22] A. J. Hudspeth, "How the ear's works work: mechanoelectrical transduction and amplification by hair cells," Comptes Rendus Biologies, vol. 328, no. 2, pp. 155-162, 2005.

[23] A. J. Hudspeth, Y. Choe, A. D. Mehta, and P. Martin, "Putting ion channels to work: mechanoelectrical transduction, adaptation, and amplification by hair cells," Proceedings of the National Academy of Sciences of the United States of America, vol. 97, no. 22, pp. 11765-11772, 2000.

[24] C. Lee and S. Park, "A mechanical model of stereocilia that demonstrates a shift in the high-sensitivity region due to the interplay of a negative stiffness and an adaptation mechanism," Bioinspiration \& Biomimetics, vol. 7, no. 4, Article ID 046013, 2012.

[25] Q. Zheng, Y. Zhang, Y. Lei, J. Song, and Y. Xu, "Haircellinspired capacitive accelerometer with both high sensitivity and broad dynamic range," in Proceedings of the 9th IEEE Sensors Conference (SENSORS '10), pp. 1468-1473, Waikoloa, Hawaii, USA, November 2010.

[26] C. Smith, A. Villanueva, and S. Priya, "Aurelia aurita bioinspired tilt sensor," Smart Materials and Structures, vol. 21, no. 10, Article ID 105015, 2012.

[27] L. J. Liu and Y. Lei, "Mechanism of a hair cell bioinspired sensor with ultrasensitivity to weak and low frequency vibration signals," International Journal of Distributed Sensor Networks, vol. 2013, Article ID 278151, 10 pages, 2013.

[28] H. Allag, J.-P. Yonnet, and M. E. H. Latreche, "3D analytical calculation of forces between linear halbach-type permanentmagnet arrays," in Proceedings of the 8th International Symposium on Advanced Electromechanical Motion Systems and Electric Drives Joint Symposium (ELECTROMOTION '09), pp. 1-6, July 2009. 


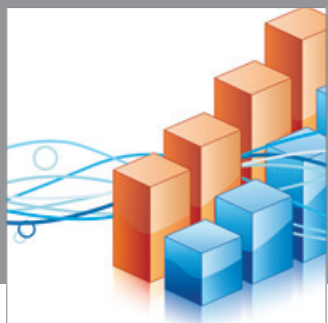

Advances in

Operations Research

mansans

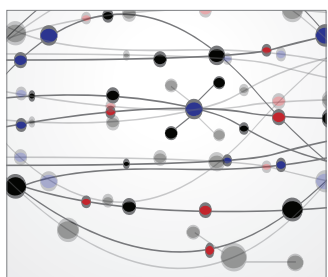

The Scientific World Journal
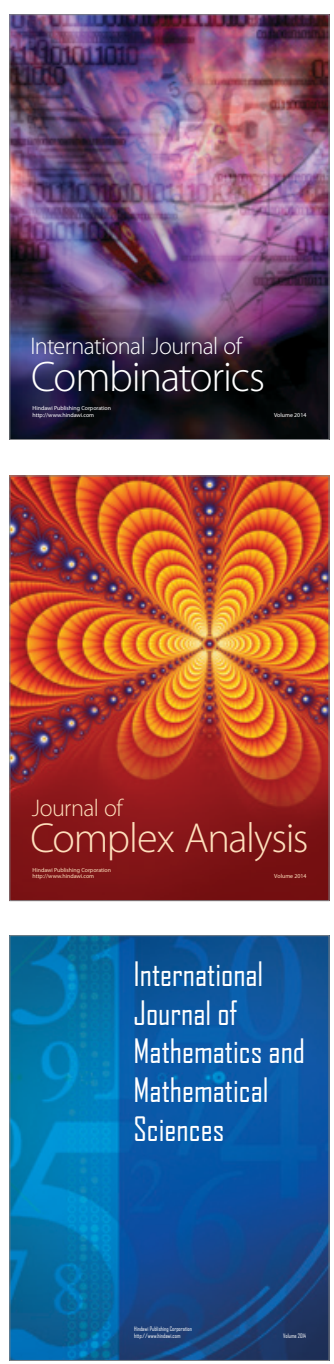
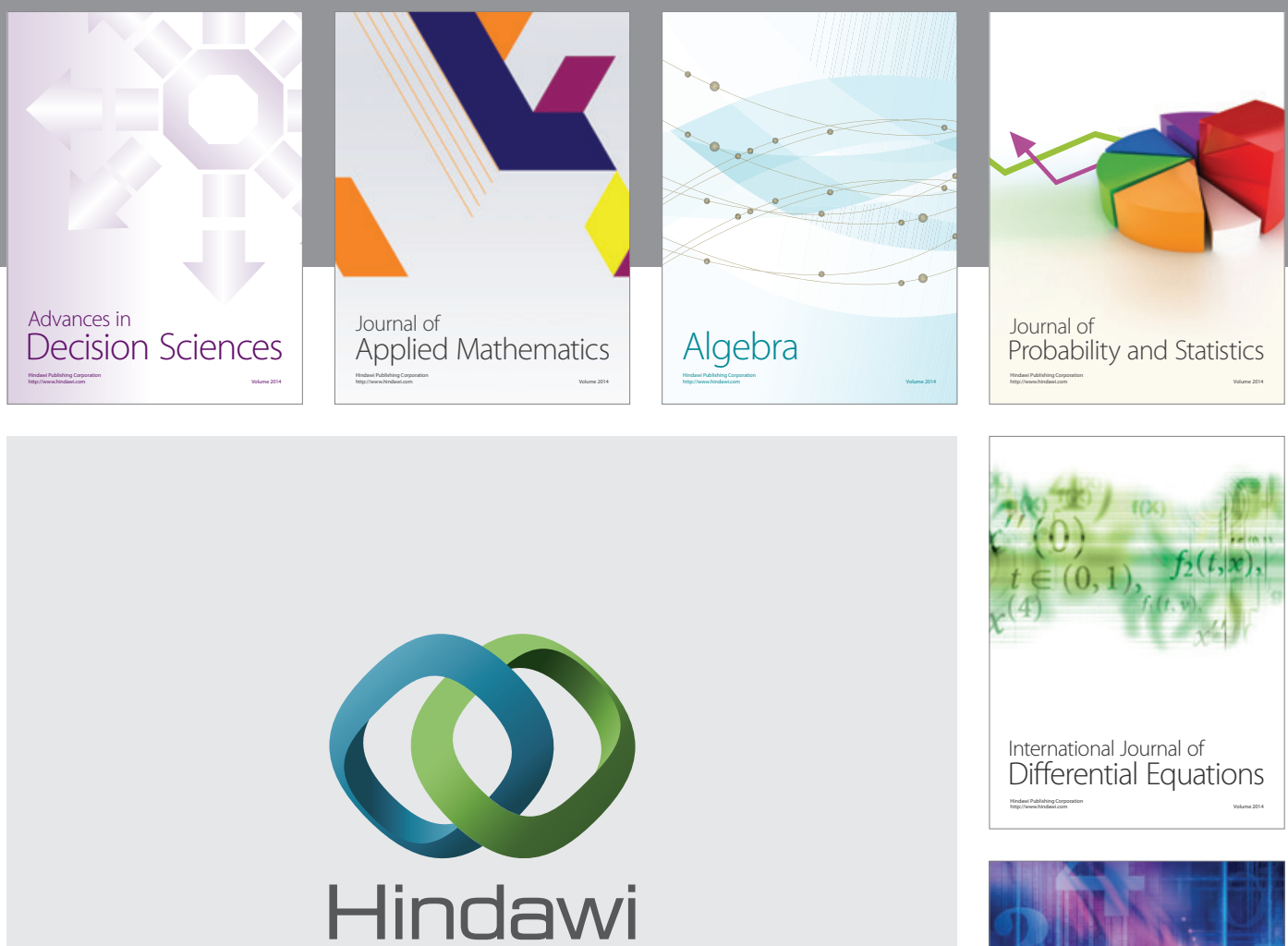

Submit your manuscripts at http://www.hindawi.com
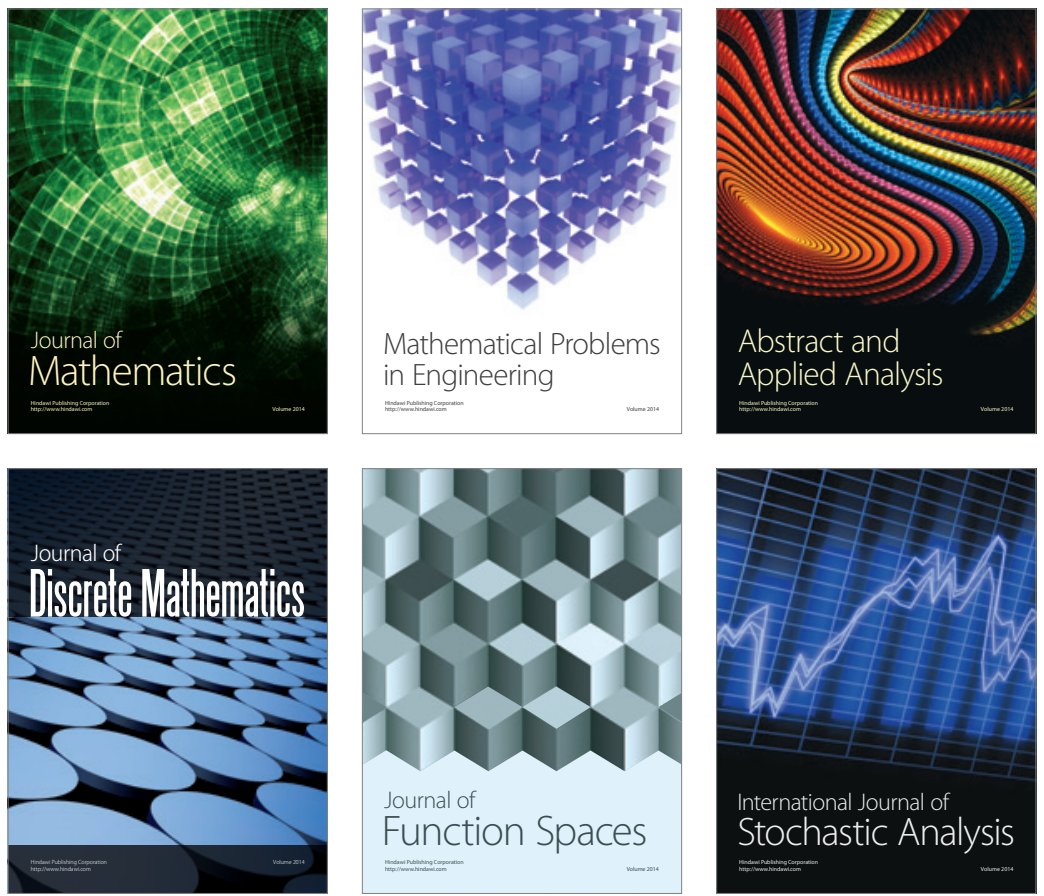

Journal of

Function Spaces

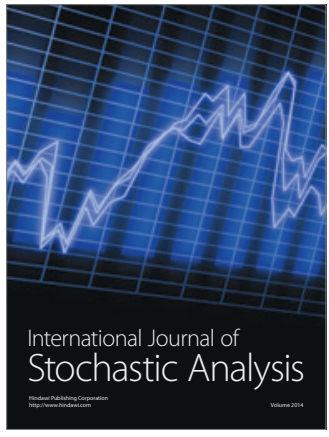

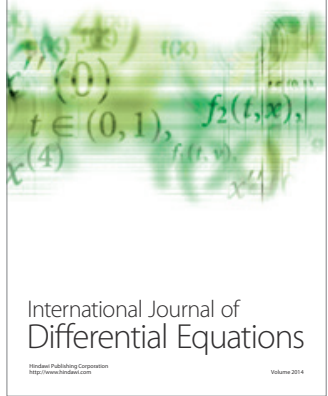
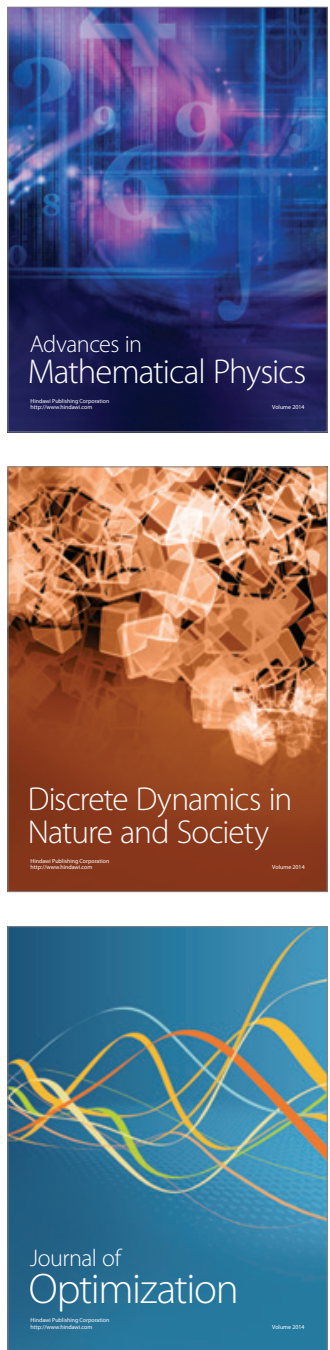